\title{
One-pot chemistry: Alkyne-assisted CNT growth enables in situ functionalization
}

\author{
Jennifer Carpena-Núñez ${ }^{\star} \odot$, Rahul Rao $\odot$, and Benji Maruyama $\odot$
}

\author{
Submitted February 8, 2021; Accepted February 26, 2021
}

$\mathrm{C}$ urrent and future global trends have propelled the materials community toward the development of green materials and technology. For instance, at the plenary session of the MRS 2020 Virtual Spring/Fall Meeting \& Exhibit, IBM presented their 5-in-5 list: five ways in which our lives and, more specifically, the materials community, will change the future within the next 5 years. ${ }^{1}$ The 5 -in- 5 predicts technological advancements that will, along with three other predictions, help achieve (1) the efficient capture and transformation of $\mathrm{CO}_{2}$ and (2) the manufacture of more sustainable materials. Meanwhile, the oil industry (e.g., Shell Oil Company and others) has released similar $\mathrm{CO}_{2}$ capture and avoidance initiatives to help battle climate change threats posed by rising carbon emissions. ${ }^{2}$ Likewise, academia, government, and startup companies (e.g., SkyNano Technologies and Rice University's Carbon $\mathrm{Hub}$ ) are pursuing $\mathrm{CO}_{2}$ capture schemes, along with strategies for the co-production of clean hydrogen and solid carbon from the pyrolysis of methane. ${ }^{3,4}$ These are only a few examples of recent thrusts driving the community toward sustainable materials and green chemistry processes.

The work by Johnson et al. ${ }^{5}$ and the Plata group at the Massachusetts Institute of Technology is steered by similar green chemistry thrusts, aiming to establish protocols for the synthesis and post-synthesis processing of carbon nanotubes (CNTs) that reduce their footprint on the environment. CNTs have been proposed as conduits for combating climate change as by-products of $\mathrm{CO}_{2}$ conversion and sequestration and as by-products of methane pyrolysis in the SkyNano and Carbon Hub initiatives mentioned earlier. ${ }^{3,4}$ These superb materials have the potential to be some of the most impactful materials of this century, carrying the promise of unparalleled properties. $^{6}$

The basic inputs for the synthesis of CNTs are similar to those used in the production of carbon black and carbon fiber. Typically, the process uses a hydrocarbon as a precursor, a transition metal catalyst (which are inexpensive, earth-abundant, and non-strategic), and a source of heat. ${ }^{6-8}$ Yet current CNT synthesis is far from being scalable and costeffective; at a price range of $\sim$ USD $85,000-300,000$ per metric ton (dependent on the quality), it out-costs carbon black (sold at $\sim$ USD1,000 per metric ton) by a factor of $>85$ and carbon fiber by a factor of $>4$. CNT technology has been impeded by synthesis issues and labor-intensive processing (e.g., functionalization and integration into composites) to completed articles: low yields, high impurity content, batch-to-batch variability, poor structural and functional control, and dispersion, among others. ${ }^{6}$ Should these challenges be overcome, CNT production could be scaled to millions of tons per year to meet the demands for carbon black (produced at $>10$ million tons per year), and possibly displace larger markets (e.g., copper and aluminum) in conductive and structural applications. ${ }^{3,9}$

Among the many processing challenges, surface functionalization represents one of the hardest problems to tackle- - a challenge that Johnson et al. ${ }^{5}$ take on with their work. Functionalization lends CNTs several advantages over their pristine counterparts: It allows them to become miscible in solvents or matrices, improves dispersion, and promotes their binding to other chemical species, which is the basis of key applications, such as chemical- and bio-sensing. Current functionalization strategies, which yield $\sim 10 \%$ surface coverage, often make use of secondary solvent-processing; these secondary processes can degrade the CNT properties, generate toxic waste streams (e.g., acid treatments), and be time consuming and costly.

To circumvent the negative traits associated with postsynthesis surface functionalization, Johnson et al..$^{5}$ propose a universal oxygen-functionalization strategy that leverages green chemistry principles and one-pot synthesis/processing protocols. Previously, Plata et al. reported on a polymerization-like growth mechanism, where the incorporation of $\mathrm{C}_{2}-\mathrm{C}_{4}$ subunits (e.g., alkynes) and an electron exchange process (i.e., reduction/oxidation) provide the means for CNT growth (see Figure 1). ${ }^{10}$ In the MRS Bulletin Impact article, ${ }^{5}$ the authors

Jennifer Carpena-Núñez, Materials and Manufacturing Directorate, Air Force Research Laboratory, Wright-Patterson Air Force Base, USA; UES, Inc., USA; jennifer.carpena.ctr@afresearchlab. com

Rahul Rao, Materials and Manufacturing Directorate, Air Force Research Laboratory, Wright-Patterson Air Force Base, USA

Benji Maruyama, Materials and Manufacturing Directorate, Air Force Research Laboratory, Wright-Patterson Air Force Base, USA

${ }^{*}$ Corresponding author

doi:10.1557/s43577-021-00080-w 


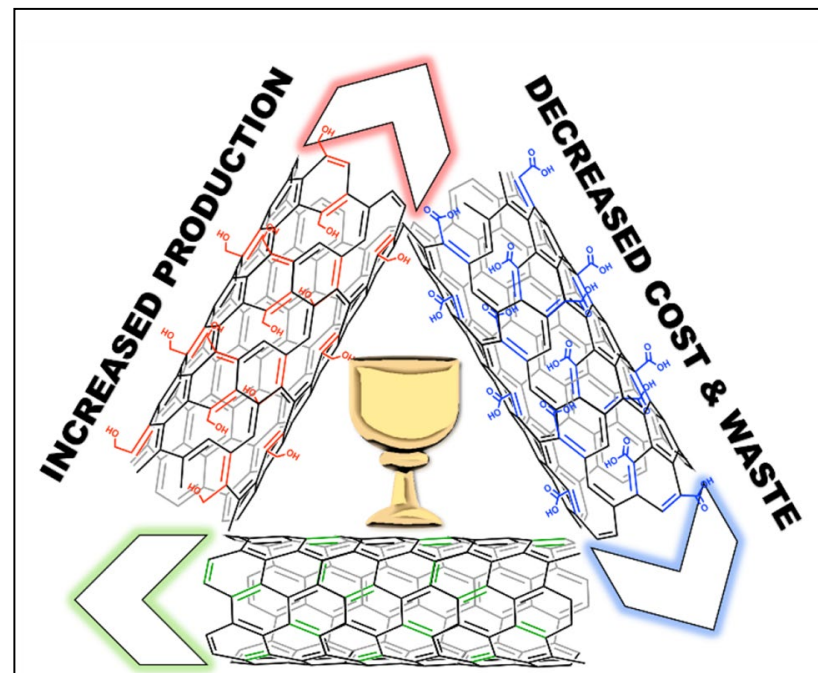

EFFICIENT \& SUSTAINABLE

Figure 1. The holy grail of carbon nanotube (CNT) synthesis and processing: increased production, reduced cost and waste streams, improved quality and efficiency, and sustainability. Johnson et al. ${ }^{5}$ work toward these goals by coupling CNT growth and functionalization using alkyne-assisted chemistry. The schematic depicts the CNT structures that Johnson et al..$^{5}$ propose are formed during the in situ functionalization protocol: propargyl alcohol (red), propiolic acid (blue), and acetylene (green). ${ }^{5}$ Adapted from Figure $\mathrm{S} 1$ in Reference 5.

expand on this concept and demonstrate that the same procedure can be used to incorporate oxygen-containing species without the need for additional processing (i.e., during the formation of said lattice). The authors demonstrate that (1) CNTs are derived from alkyne precursors, namely propargyl alcohol and propiolic acid, and that these oxygen-containing alkyne precursors also lead to (2) subtle incorporations of carboxyl and hydroxyl functionalities on the CNTs. Beyond proof-ofconcept, the authors elucidate the role of alkyne precursors in functionalizing the CNTs. They found that these alkynes shed their oxygen groups before or during their incorporation into the growing lattice, resulting in only a small degree of functionalization ( $\sim 1 \%$ compared to the $\sim 10 \%$ obtained from conventional methods). Furthermore, analyses suggested that functionalization is limited by (1) the formation of a reactive atmosphere high in methylacetylene in the case of propargyl alcohol and (2) the thermal degradation to acetylene and $\mathrm{CO}_{2}$ in the case of propiolic acid. The authors also demonstrate that this same shedding mechanism, and therefore delivery of bare alkynes, leads to enhanced catalytic lifetimes.

While the in situ strategy could effectively combine growth and functionalization into a single step, it is far from being technology-ready. Further characterization of the functionalities is needed, as are insights into the mechanisms responsible for in situ functionalization during hightemperature CNT synthesis. Aware of this, Johnson and coauthors ${ }^{5}$ effectively delineate the limitations of their process, provide hypotheses for hindering mechanisms, and propose future research directions. There is great promise from the work of Johnson et al. to reduce waste streams, reduce cost, speed up processing, and improve properties. If successfully optimized, this green chemistry approach may offer CNT researchers a grand opportunity: the pursuit of sustainable and universal one-pot synthesis protocols that enable controlled functionalization of CNTs.

\section{References}

1. https://www.research.ibm.com/5-in-5/.

2. https://www.shell.com/sustainability/environment/climate-change/carbon-captu re-and-storage-projects.html.

3. https://carbonhub.rice.edu/.

4. https://www.skynanotechnologies.com/.

5. E.P. Johnson, W. Shi, D.L. Plata, MRS Bull. Impact 46 (2021), forthcoming.

6. R. Rao, C.L. Pint, A.E. Islam, R.S. Weatherup, S. Hofmann, E.R. Meshot, F. Wu, C. Zhou, N. Dee, P.B. Amama, J. Carpena-Nunez, W. Shi, D.L. Plata, E.S. Penev, B.I. Yakobson, P.B. Balbuena, C. Bichara, D.N. Futaba, S. Noda, H. Shin, K.S. Kim, B. Simard, F. Mirri, M. Pasquali, F. Fornasiero, E.I. Kauppinen, M. Arnold, B.A. Cola, P. Nikolaev, S. Arepalli, H.M. Cheng, D.N. Zakharov, E.A. Stach, J. Zhang, F. Wei, M. Terrones, D.B. Geohegan, B. Maruyama, S. Maruyama, Y. Li, W.W. Adams, A.J. Hart, Carbon nanotubes and related nanomaterials: Critical advances and challenges for synthesis toward mainstream commercial applications. ACS Nano 12(12), 11756 (2018)

7. J. Carpena-Nunez, J.A. Boscoboinik, S. Saber, R. Rao, J.Q. Zhong, M.R. Maschmann, P.R. Kidambi, N.T. Dee, D.N. Zakharov, A.J. Hart, E.A. Stach, B. Maruyama, Isolating the roles of hydrogen exposure and trace carbon contamination on the formation of active catalyst populations for carbon nanotube growth. ACS Nano 13(8), 8736 (2019)

8. J. Carpena-Nunez, R. Rao, D. Kim, K.V. Bets, D.N. Zakharov, J.A. Boscoboinik, E.A. Stach, B.I. Yakobson, M. Tsapatsis, D. Stacchiola, B. Maruyama, Zeolite nanosheets stabilize catalyst particles to promote the frowth of thermodynamically unfavorable, small-diameter carbon nanotubes. Small 16(38), e2002120 (2020)

9. N. Behabtu, C.C. Young, D.E. Tsentalovich, O. Kleinerman, X. Wang, A.W. Ma, E.A. Bengio, R.F. ter Waarbeek, J.J. de Jong, R.E. Hoogerwerf, S.B. Fairchild, J.B. Ferguson, B. Maruyama, J. Kono, Y. Talmon, Y. Cohen, M.J. Otto, M. Pasquali, Strong, light, multifunctional fibers of carbon nanotubes with ultrahigh conductivity. Science 339(6116), 182 (2013)

10. D.L. Plata, E.R. Meshot, C.M. Reddy, A.J. Hart, P.M. Gschwend, Multiple alkynes react with ethylene to enhance carbon nanotube synthesis, suggesting a polymerization-like formation mechanism. ACS Nano 4(12), 7185 (2010) 